\title{
Les restaurations de rivières sur Internet : premier bilan
}

\author{
Bertrand Morandi, Hervé Piégay \\ Géographes, Université de Lyon, UMR5600 EVS, CNRS, Site ENS de Lyon, 69342 Lyon cedex 07, France
}

L'outil Internet est aujourd'hui complètement intégré au monde de la recherche, dont il a profondément modifié les pratiques. Dans la recherche finalisée, les chercheurs ont appris à le maîtriser pour recueillir de l'information à travers des enquêtes, communiquer, diffuser leurs résultats, etc. L'originalité de cet article est d'analyser les pratiques de restauration de cours d'eau et la mise en œuvre d'une politique publique, la directive-cadre européenne sur l'eau, à partir de l'information mise en ligne par les acteurs de la gestion des rivières.

La Rédaction

\section{Mots-clés :}

restauration écologique ; hydrosystèmes fluviaux ; recensement Internet ; géographie ; France

\section{Keywords:}

ecological restoration; fluvial hydrosystems; Internet census; geography; France

\begin{abstract}
Résumé - En érigeant le «bon état écologique» des rivières en objectif à atteindre, la directive-cadre européenne sur l'eau oblige à questionner les pratiques actuelles et passées dans le domaine de la restauration. Un premier recensement national a été réalisé via Internet et a permis d'identifier 480 actions déclarées comme relevant du domaine de la restauration de rivières. Les résultats conduisent à formuler plusieurs constats : 1 . Le terme de restauration a un sens qui diffère selon les acteurs, renvoyant indifféremment à une approche hydraulique, écologique ou piscicole; 2 . Des différences régionales sont observées tant en termes de types d'action que de fréquence; 3 . Les conceptions et les pratiques évoluent peu sur la période considérée par l'analyse (1985-2009) ; 4. L'action est davantage valorisée que son résultat ; 5. Enfin, les résultats du recensement montrent que la diffusion de l'information est significative dans le domaine, mais que les stratégies de communication varient en fonction des communicants.
\end{abstract}

\begin{abstract}
River restoration experiences on the web: operational outcomes and perspectives. The EU Water Framework Directive (WFD) set the "good ecological status" of rivers as an objective to be reached before 2015. Monitoring, evaluation and feedback are therefore necessary. This paper highlights results from a first Internet French census which identified 480 initiatives declared as restoration actions. Results show that: 1 . The definition of restoration is as yet neither clear nor univocal. Policies and competent stakeholders do not suffice to produce effective ecological restoration. Nineteenth century hydraulic engineering approaches are still being implemented. In many cases, objectives focus on security and free flow. There are confusions between river restoration, maintenance and management; 2. Geographic differences about quantity and quality of restoration are obvious but are not fully understood; 3 . Inversely, restoration conceptions and practices show little change over the period analysed in the study (1985-2009); 4. Limitations in river restoration assessment are highlighted. There is no feedback on ecological and social gains in most actions. On the Internet, actions have priority over restoration results; 5 . The Internet promotes exchanges and work on action and communication strategies in river restoration issues. As such it is particularly valuable in the operational and scientific fields where data sharing is an important factor.
\end{abstract}

\section{Introduction}

La restauration écologique est aujourd'hui un concept à la mode qui fait l'objet d'un véritable engouement scientifique (Le Floc'h et Aronson, 1995). Dans le domaine des milieux aquatiques, et avec la mise en œuvre de la directive-cadre européenne sur l'eau (DCE), elle est devenue un objectif opérationnel de premier ordre. La

Auteur correspondant : B. Morandi,

bertrand.morandi@ens-lyon.fr restauration apparaît en effet comme un moyen privilégié pour atteindre le «bon état écologique» des cours d'eau. Dans ce contexte, l'enjeu du présent article ${ }^{1}$ est de proposer un premier bilan français en termes d'action, d'évaluation et de communication dans le domaine de la restauration de rivières. Il s'agit également de faire émerger des questions de recherche jusqu'ici peu explorées à

\footnotetext{
${ }^{1}$ Ces travaux s'inscrivent dans le cadre de la zone atelier Bassin du Rhône et du projet européen IWRM/Net FORECASTER (2008-2010).
} 
l'échelle nationale, en adoptant un positionnement analytique global. La contrainte réglementaire est en effet de réparer, mais aussi de démontrer que les réparations sont efficaces, appliquant une obligation de résultats et non plus de moyens, et impliquant de fait un débat sur l'évaluation (Sachon et al., 2003) qui occupe aujourd'hui une place centrale dans la réflexion sur la restauration (Jenkinson et al., 2006; Kondolf et al., 2007). Les recensements américains (Bernhardt et al., 2007) lancés depuis le début des années 2000 ont tenté de pallier le manque $\mathrm{d}$ 'informations constaté. Ils ont permis d'activer la sphère scientifique par des retours d'expériences, dont les premières conclusions soulignent le manque de lisibilité de nombreuses actions. En France, le constat est sensiblement le même puisque aucun bilan national n'a jusqu'ici été dressé. Les questions sont encore nombreuses concernant le concept même de restauration et le premier débat est sémantique. Qu'est-ce qu'une action de restauration? À partir de quelle(s) définition(s) travaillons-nous? Le concept comme la discipline qui le prend pour objet sont en construction (National Research Council, 1992; Cairns et Heckmann, 1996). Les approches en sont plurielles, des conceptions hydrauliciennes à la réflexion écosystémique, en passant par les sciences sociales (Palmer et Bernhardt, 2006) ; les déclinaisons en sont multiples, de la restauration d'un état originel à la réhabilitation d'un «bon état écologique »(Bradshaw, 1996), et les actions mises en œuvre sont riches de diversité (reméandrage, recharge sédimentaire, relèvement des débits, etc.). Le second débat porte sur l'indication. Comment évaluer les réussites et les échecs ? Comment quantifier les gains? Le besoin de réponse s'exprime scientifiquement mais aussi opérationnellement, ne serait-ce que pour savoir quelles actions doivent être reproduites. Enfin, il y a un fort enjeu de communication, qu'elle se fasse entre scientifiques, entre recherche et sphère opérationnelle ou entre acteurs de l'eau et grand public (Malavoi et Adam, 2007; Piégay et al., 2009).

Ce dernier point a ainsi motivé le choix du médium Internet pour conduire un recensement national au début de l'année 2009. Outre la facilité d'accès aux données, l'outil permet de conduire une réflexion sur les stratégies de diffusion.

Plusieurs hypothèses ont été formulées : 1 . Le terme de restauration n'est pas univoque et regroupe une diversité d'actions parfois contradictoires. Un débat sémantique est ouvert (Barnaud, 1995) ; 2. Une variabilité régionale des actions de restauration est ensuite supposée. Certaines régions seraient plus portées que d'autres à communiquer ou à agir. L'Alsace, par exemple, proche de l'Allemagne où la conscience environnementale est plus développée (Le Lay et al., 2008), serait-elle plus dynamique en matière de restauration? 3. Les nouvelles contraintes réglementaires et les nouvelles préoccupations environnementales se traduisent sans doute dans le domaine de la restauration de rivière. Une évolution temporelle des conceptions devrait être observée (Dufour et Piégay, 2009) ; 4. La DCE oblige à des résultats. Savoir quels sont les bénéfices sociétaux de ces actions devient un enjeu et disposer de retours d'expériences, une nécessité. Quelle est l'importance accordée à l'évaluation? Parle-t-on de ce que l'on fait ou des résultats? 5. Cela nous conduit à la dernière hypothèse, portant plus spécifiquement sur la question de la communication. Qui fait quoi ? Qui communique quoi ? Internet est un espace permettant de faire connaître les actions et de valoriser l'image des structures engagées. En fonction de la nature des opérations et du type d'acteurs, on peut donc supposer des différences d'implication.

\section{Méthodes de recensement}

Tout travail de recensement doit répondre à des principes méthodologiques précisément définis. Deux étapes sont ainsi identifiées : construire conceptuellement l'objet d'étude ; définir des méthodes pratiques de recherche de l'information et d'utilisation du médium Internet.

\section{Définition conceptuelle de l'objet d'étude et identification des variables}

Le recensement ne repose pas sur un positionnement a priori, mais sur une démarche empirique fondée sur la réalité des actions. Ont ainsi été considérées les actions déclarées par les opérateurs, les élus ou les scientifiques comme relevant du domaine de la restauration de rivière. Le concept est ancré dans la pratique et il semblait indispensable d'alimenter la réflexion théorique en partant de la manière dont est conçue et réalisée l'action. Une telle démarche ne doit pas pour autant conduire à considérer de manière irraisonnée toutes les opérations du moment que le terme de restauration est employé. Les travaux doivent porter sur l'hydrosystème et non sur des infrastructures annexes (moulins, ponts, etc.). Les problématiques de franchissement piscicole et les actions de réintroduction animale n'ont pas été considérées non plus. L'échelle globale de travail, quant à elle, est nationale et les informations proviennent d'Internet. L'approche est, pour l'essentiel, quantitative. Ont été privilégiés à la fois l'action, ses cadres et ses modes d'évaluation (Tab. 1).

\section{Protocoles d'utilisation du médium Internet}

L'usage d'Internet suppose d'en mesurer les limites et de les prendre en compte à la fois dans la définition des protocoles de recensement et dans l'analyse de l'information acquise. La question de la temporalité de l'information est primordiale, car l'utilisation de la Toile s'est 
Tableau 1. Grille de recensement simplifiée.

\begin{tabular}{l}
\hline Identification du site restauré \\
\hline Nom du cours d'eau \\
Date de l'action \\
Linéaire / surface restaurée \\
Nom du site / de l'opération \\
Localisation administrative : région(s), \\
département(s), commune(s) \\
\hline Définition de l'action de restauration \\
\hline Pressions \\
Objectifs de restauration \\
Descriptifs des travaux \\
Coût de l'action \\
Maîtrise d'ouvrage \\
Structures associées / partenaires \\
\hline Éléments d'évaluation \\
\hline Suivi scientifique avant restauration / état initial \\
États de références \\
Suivi scientifique après restauration \\
Suivi scientifique sur un site témoin \\
Suivi technique \\
\hline Métadonnées \\
\hline Sources bibliographiques et sitographiques \\
Liens Internet \\
\hline
\end{tabular}

vulgarisée récemment, ce qui limite les analyses chronologiques. La nature et la fiabilité de l'information sont ensuite à questionner. Internet constitue un simple médium et il est important de porter un regard critique sur les données qui y sont présentées. La mise en ligne relève d'une stratégie volontaire de la part du communicant qu'il est primordial d'identifier (Andrieu, 1998). Par ailleurs, l'information est souvent lacunaire et le recensement ne peut donc être exhaustif. Il porte sur ce qui est présent sur Internet et non sur toutes les opérations effectivement réalisées. Enfin, les principes de fonctionnement des moteurs de recherche sont discutables à plus d'un titre (Maniez, 2008) et il est important d'utiliser des protocoles précisément définis. Le recensement a ainsi été effectué selon des démarches complémentaires. La première recherche a consisté en une exploration systématique des sites institutionnels et des grandes plateformes Internet produites par les structures compétentes: agences de l'eau, Office national de l'eau et des milieux aquatiques (ONEMA), syndicats en charge des schémas d'aménagement et de gestion des eaux (SAGE) ou des contrats de rivières (CR), Fédération nationale pour la pêche en France (FNPF) et ses fédérations départementales ou associations agréées pour la pêche et la protection du milieu aquatique (FDPPMA et AAPPMA). Le système d'information sur l'eau et notamment la plateforme GEST'EAU ont constitué des supports précieux. Le manque d'information constaté pour certaines opéra- tions a nécessité une recherche complémentaire. Moins exhaustive, cette dernière a été fondée sur le principe de «navigation » propre à Internet. Elle a utilisé le moteur de recherche Google et un système de requêtes par mots-clés. Les requêtes, réalisées pour une liste de 144 cours d'eau susceptibles d'avoir fait l'objet d'une restauration, ont permis d'éviter toute confusion lexicale, généralement courante dans l'utilisation des moteurs de recherche. Une exploration systématique des liens présents sur les deux premières pages de résultats a ensuite été réalisée. Cette démarche est destinée à cadrer la recherche plus qu'à la préciser, puisque les premiers résultats ne sont pas toujours les plus intéressants (Boni et Stemart, 2008).

\section{Les actions portées à connaissance sur Internet}

\section{Nature de l'information}

Au total, ce sont 480 actions de restauration qui ont été localisées à l'échelle du territoire métropolitain. L'identification du lieu précis de l'opération n'a pas été possible pour $9 \%$ d'entre elles et la localisation a alors été faite au niveau du «cours d'eau ». Les données sont également lacunaires pour certaines variables en fonction de l'action considérée. Les dates sont disponibles pour $70 \%$ des actions, la maîtrise d'ouvrage et le descriptif des travaux pour respectivement $67 \%$ et $96 \%$ d'entre elles. Enfin, pour certaines variables (pressions, objectifs et éléments d'évaluation), les valeurs nulles ont été prises en compte dans les traitements car elles peuvent devenir significatives des pratiques de restauration.

Les mesures mises en œuvre ont fait l'objet d'une classification empirique a posteriori, à partir du descriptif des travaux (Tab. 2). Cela permet d'identifier des mesures différentes (rang 2), appartenant à plusieurs types de restauration (rang 1) et traduisant des conceptions diverses de l'action. La méthode de définition n'est toutefois pas sans limite dans la mesure où Internet n'autorise qu'à travailler indirectement sur les conceptions. Elle permet de distinguer les actions écologiques, centrées sur le fonctionnement du système, et les interventions piscicoles, qui portent sur la ressource ou la gestion d'espèces particulières. Elle intègre également le concept de restauration hydraulique. À l'échelle nationale, on note une dominance de ce type de restauration, puisque $49 \%$ des actions intègrent une dimension hydraulique, validant ainsi notre hypothèse de départ. Les actions écologiques et piscicoles représentent respectivement $42 \%$ et $27 \%$ des interventions, et $11 \%$ des actions hydrauliques sont couplées à des mesures écologiques. Aux États-Unis, le constat est sensiblement le même puisque le terme de restauration recouvre également des réalités différentes, avec une forte proportion de mesures 
Tableau 2. Classification des actions en fonction du type de restauration et des mesures mises en œuvre. Figurent entre crochets les codes utilisés dans les traitements des données.

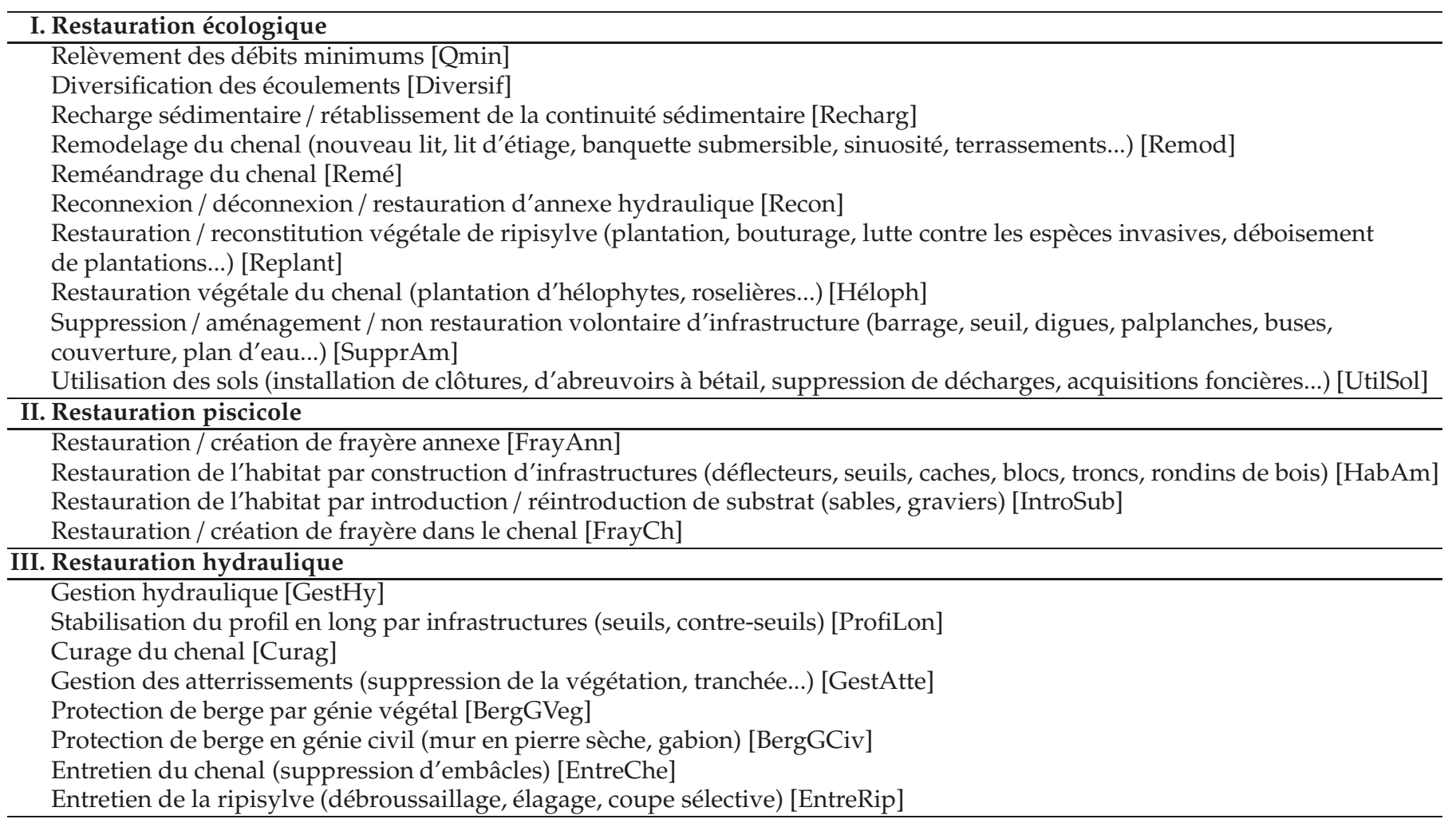

hydrauliques (stabilisation de berge, gestion des eaux d'orages) et de mesures d'entretien (gestion de ripisylve) [Bernhardt et al., 2007].

Si l'on s'intéresse ensuite au détail des mesures (Fig. 1), on peut observer que les travaux d'entretien et de protection de berge représentent respectivement $23 \%$ et $16 \%$ des mesures appliquées. Certaines mesures écologiques et piscicoles sont toutefois amplement répandues, tels l'aménagement d'habitats (12\%) et la reconnexion d'annexes $(11 \%)$. D'autres apparaissent comme des actions expérimentales, mises en œuvre sur des sites pilotes, ou comme des mesures génériques qui peuvent échapper à la méthode de recensement : c'est le cas du relèvement des débits minimums (5 actions, soit $0,7 \%$ du total des mesures). Parallèlement, on observe une faible représentation de certaines pratiques qui ont pourtant dominé les modes de gestion dans le domaine de l'eau : longtemps intégré aux actions de restauration et d'entretien, le curage ne représente plus que $0,8 \%$ des mesures.

\section{Répartition spatiale des actions de restauration}

La répartition spatiale des actions n'est pas uniforme (Fig. 2). Certaines régions apparaissent particulièrement pauvres en matière de restauration, comme le NordPas-de-Calais $(0,8 \%)$, la Picardie $(0,2 \%)$ ou encore la
Corse $(0 \%)$. Inversement, on peut remarquer le poids de la région Rhône-Alpes, qui concentre à elle seule $44 \%$ des actions. L'absence de corrélation nette entre le nombre d'actions de restauration et le nombre de CR et de SAGE, observée à l'échelle régionale, laisse supposer que la mise en œuvre de mesures de restauration dépendrait de contextes et de besoins locaux différents. Il serait sur ce point intéressant d'analyser la répartition des actions au sein des structures, en prenant en compte les autres volets d'intervention (assainissement, pollution...). On peut aussi estimer que les variations portent sur la communication et non sur l'action. Certains syndicats communiquent moins ou sur des actions dont la nature n'est pas la même (protection des biens et des personnes, assainissement, etc.). On peut enfin proposer une explication sémantique. La communication se fait relativement à une définition de la restauration propre à chaque structure. Au-delà des structures et de la question des stratégies de communication, il est possible de formuler d'autres hypothèses : le défaut d'action serait lié à une moindre dégradation des cours d'eau dans certaines régions; tous les types de dégradation n'auraient pas été considérés dans le recensement. Les réponses aux dégradations physico-chimiques de l'eau peuvent, pour certains bassins, avoir été favorisées au détriment des actions d'un autre type (par exemple pour le bassin 


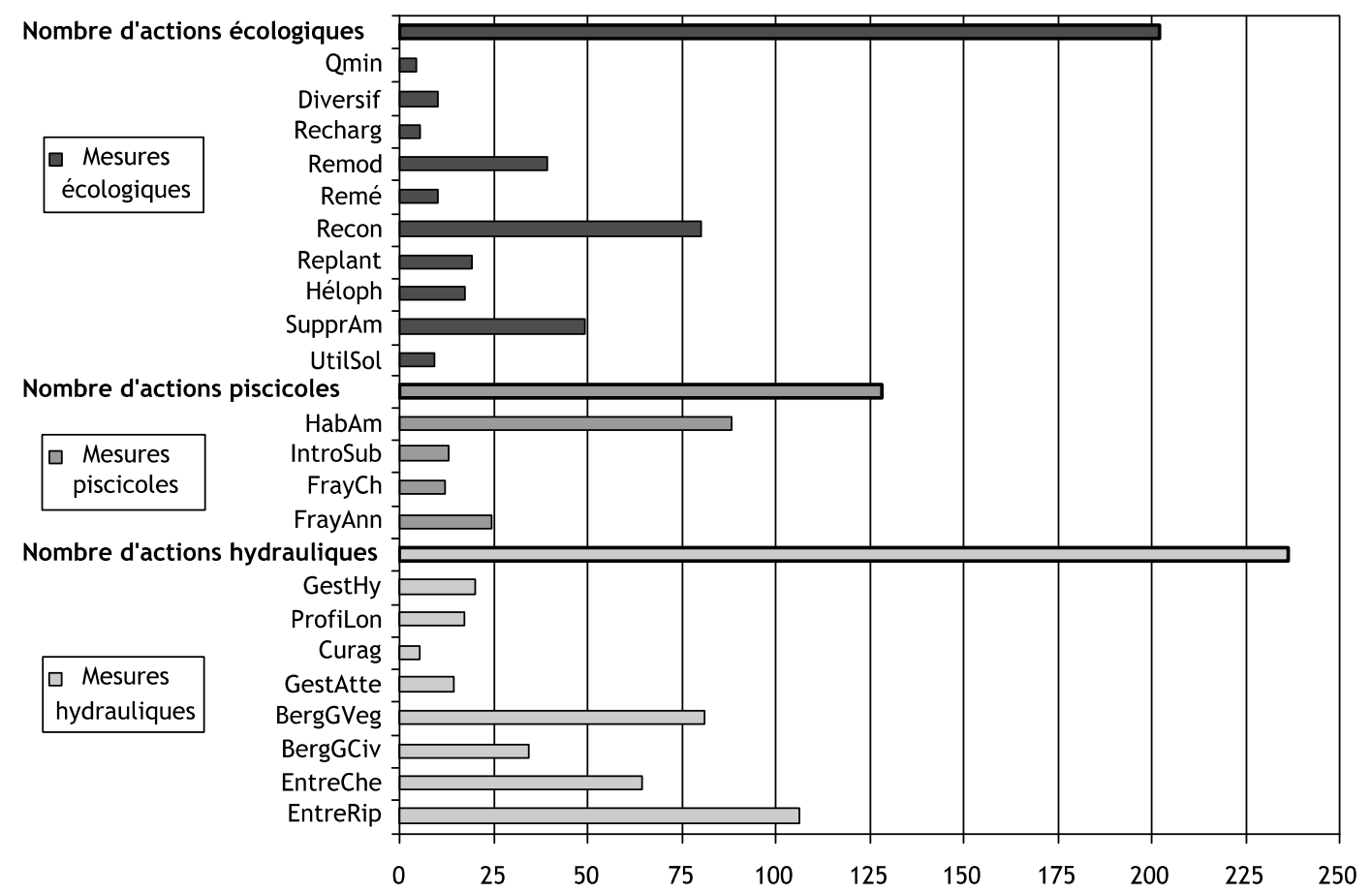

Fig. 1. Nombre de mesures de restauration mises en œuvre dans les 480 actions recensées (voir Tab. 2 pour les codes).

Artois-Picardie). Inversement, les cours d'eau alpins ont été soumis à différentes formes de pressions physiques (Habersack et Piégay, 2007), ce qui apparaît comme un élément permettant de comprendre la densité des actions rhônalpines.

La disparité spatiale n'est pas uniquement quantitative; elle intègre aussi les différents types de restauration. La répartition des mesures peut s'expliquer par le contexte géographique et les rapports historiques que les sociétés entretiennent avec les rivières. Ceux-ci peuvent permettre de comprendre l'ancrage de certaines actions et la difficulté d'évolution des conceptions dans des espaces où la sécurité est prioritaire, comme dans les régions méditerranéennes. Ainsi, $68 \%$ des actions conduites en Languedoc-Roussillon mettent en œuvre des mesures hydrauliques, ce qui est très supérieur au pourcentage national. La région Midi-Pyrénées se situe également du côté de la politique du bon écoulement des eaux. À l'inverse, les cartes permettent de confirmer la dominance des actions de restauration écologique au sein $\mathrm{du}$ bassin hydrographique Rhin-Meuse (Fig. 2), à proximité des influences environnementalistes germaniques, confirmant ainsi notre hypothèse de départ. Par contre, le nombre important d'actions conduites au sein de la région Rhône-Alpes ne s'explique pas par la nature des mesures mises en œuvre.

\section{Évolution temporelle des formes de restauration}

La littérature scientifique insiste sur le passage d'une ancienne conception de la restauration, centrée sur la gestion locale du risque hydraulique, vers des perceptions plus écologiques de l'action contemporaine, qui s'attachent au rétablissement du fonctionnement global de l'hydrosystème (Dufour et Piégay, 2009). La classification met en exergue ces approches différentes et parfois divergentes. Les conceptions et la terminologie liées aux pratiques ingénieristes des années 1980 sont encore prégnantes sur Internet et le recensement ne permet pas de montrer d'évolution temporelle (Fig. 3). Le médium pose en outre des difficultés pour l'analyse chronologique. En effet, la vulgarisation d'Internet est récente et il est certain que les opérations les plus anciennes (avant 1990) ne sont pas mentionnées. Cela rend l'identification de tendances encore délicate. Par ailleurs, il faudrait travailler sur les dates de mise en ligne; or, celles-ci sont très rarement précisées. Les dates de début des travaux constituent néanmoins un premier support à une analyse chronologique. Le premier pic d'opérations a lieu au milieu des années 1990, sans doute lié à la mise en œuvre des schémas directeurs d'aménagement et de gestion des eaux (SDAGE) et des SAGE, mais aussi aux débuts en France des techniques de génie végétal (Lachat et al., 1994). Les opérations sont essentiellement hydrauliques, même si l'on note une première émergence des actions écologiques et piscicoles. Toutefois, la véritable mise en œuvre de telles mesures intervient au début des années 2000 et l'influence de la DCE n'y est sans doute pas étrangère. L'écart entre les actions à objectif écologique et celles à objectif piscicole se réduit et l'on note certaines années (2001 et 2005) des inversions de tendances. La diminution du nombre total d'actions à partir 

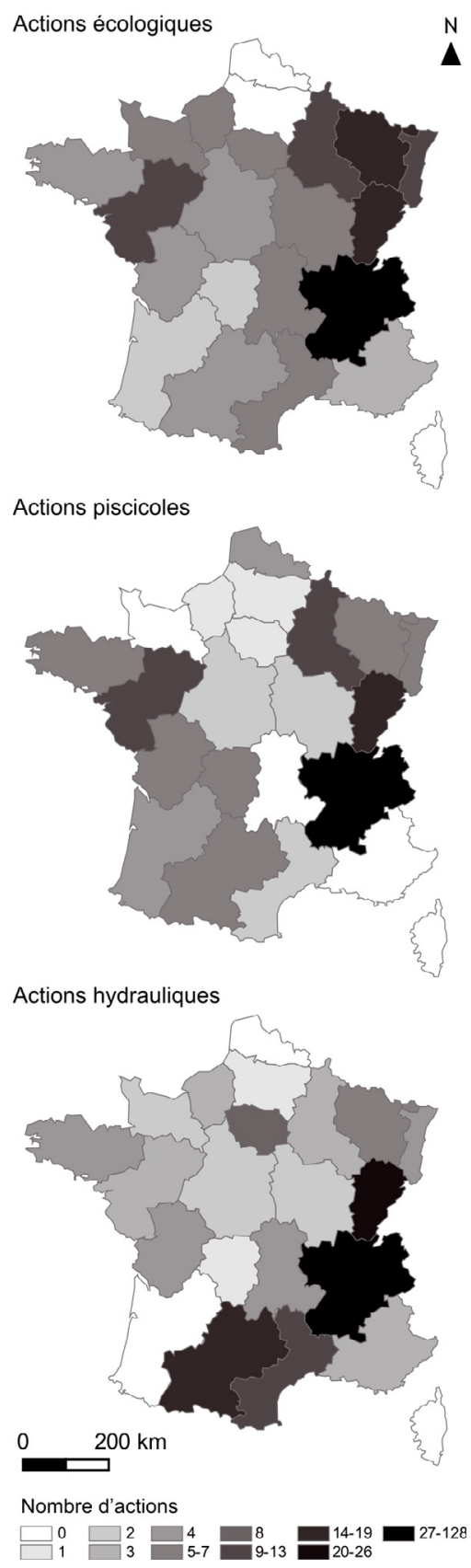

Fig. 2. Répartition nationale des actions de restauration recensées et par type de restauration : écologique, piscicole et hydraulique.

de 2006-2007 s'explique par l'absence de temps suffisant aux retours d'expériences et à la diffusion des données sur Internet. Certes, les actions écologiques et piscicoles, excepté accident annuel, n'ont pas cessé d'augmenter, mais les actions hydrauliques également, et le terme de restauration continue d'être employé indifféremment. La distinction entre la restauration écologique et ce qui est aujourd'hui qualifié d'entretien ou d'aménagement n'est pas toujours établie. L'absence de définition des pressions à l'origine des actions vient renforcer ce constat : ce manque concerne $71 \%$ des actions et empêche de fait de distinguer restauration et entretien. Il y a bien une évolution des conceptions, mais à deux vitesses. La réflexion scientifique dans le domaine de la restauration a des répercussions au niveau de l'action, mais celles-ci n'entraînent pas pour l'instant une inversion des tendances. La combinaison de mesures hydrauliques et écologiques en une même action se développe de plus en plus, sans pour autant remettre en cause le nombre de mesures uniquement hydrauliques.

\section{L'évaluation des actions de restauration}

La DCE impose l'atteinte du bon état écologique et suppose implicitement d'évaluer les actions conduites et d'en prouver l'efficacité en termes de gains écologiques à un coût acceptable. Cette phase incontournable doit être pleinement intégrée à la conception du projet (Roni et al., 2005). La manière dont les structures communiquent sur cette question est donc intéressante à analyser. Le premier constat est que les éléments disponibles sur Internet sont extrêmement limités. Ils relèvent pour la plupart de rapports mis en ligne ou de présentations scientifiques isolées. La description précise des systèmes d'évaluation reste ponctuelle et l'information se limite le plus souvent à la simple mention de l'existence d'un suivi. La question est alors de savoir si cette absence est liée à une lacune de l'évaluation ou à un manque de communication sur ce thème. Plusieurs éléments de réponse peuvent être apportés et permettent de nuancer ce constat. D'une part, Internet n'est sans doute pas le médium le plus approprié. Les stratégies de communication déjà évoquées s'adressent dans la plupart des cas à un large public, et l'information vulgarisée porte peu sur le détail des suivis scientifiques. D'autre part, le manque doit également être relativisé en tenant compte de la différence de nature des opérations. Les actions hydrauliques ou celles relevant de l'entretien sont moins suivies scientifiquement (Fig. 4).

\section{Les systèmes d'évaluation et leurs éléments structurants}

Le nombre absolu d'éléments de suivi communiqués constitue un premier indicateur. Ainsi, sur les 480 actions recensées, un élément de suivi (suivi pré- ou postrestauration, suivi d'un site témoin, suivi technique) n'est mentionné que pour 139 , soit environ $30 \%$. Ce chiffre, en apparence plus qu'honorable comparé aux résultats américains (Bernhardt et al., 2007; Bash et Ryan, 2002; Kondolf et al., 2007), ne résiste pourtant pas à l'analyse de détail. Tout d'abord, si l'on ne tient compte que des éléments de suivi scientifique, le nombre chute à 104 . Ensuite, l'élément de suivi, pris isolément, présente un intérêt limité. Il traduit l'état d'un cours d'eau en un 


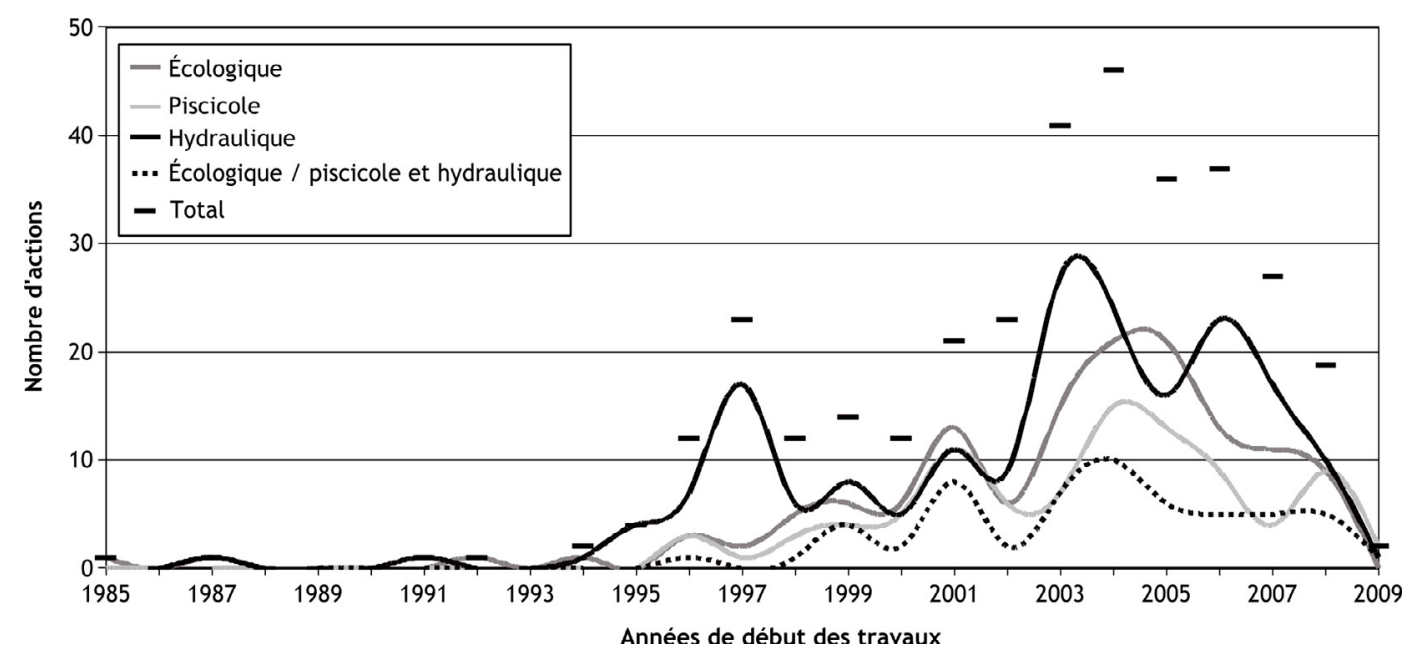

Fig. 3. Évolution temporelle du nombre d'actions en fonction du type de restauration.

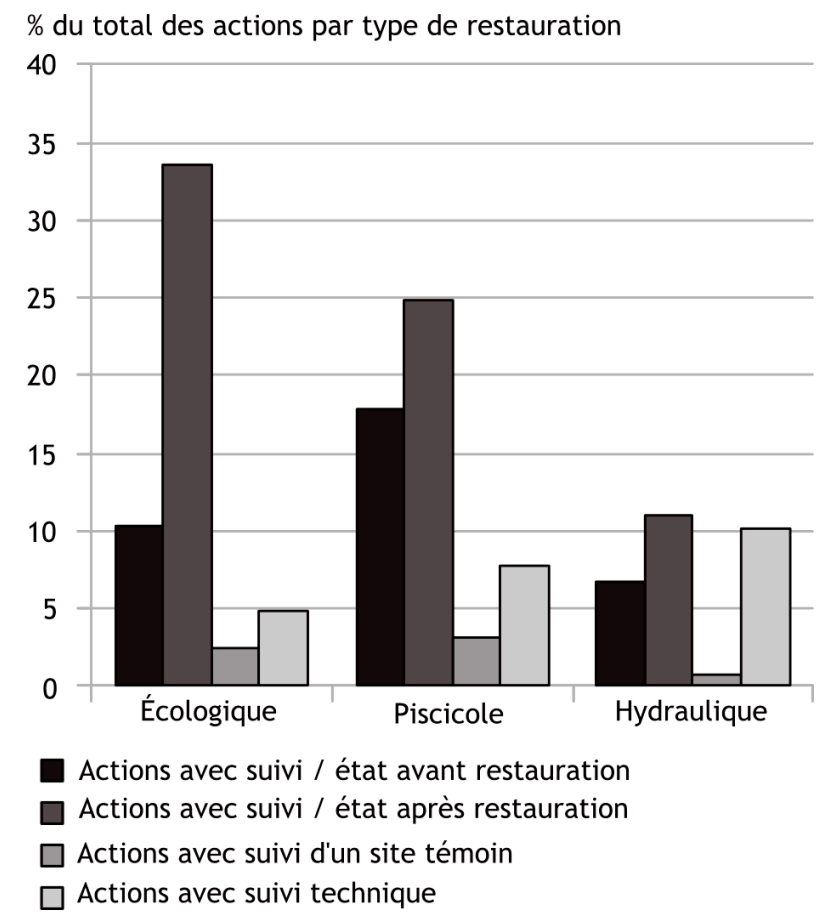

Fig. 4. Actions pour lesquelles un élément de suivi a été indiqué, en fonction du type de restauration.

instant $t$, alors que le concept de restauration est fondé sur une modification physique et biologique du compartiment traité. Plus que l'élément de suivi, ce sont donc les systèmes comparatifs d'évaluation qui intéressent, puisqu'ils permettent de travailler sur des processus. La démarche élémentaire, distinguant un état avant, un état de référence visé et un état après, est à la base de ce type d'évaluation. Or, le travail de recensement souligne que seules 35 opérations disposent d'au moins deux éléments, soit $7 \%$ du total des actions, et il n'y a que 3 cas pour lesquels états initiaux, suivis scientifiques et sites témoins sont déve- loppés conjointement. Enfin, la réutilisation des données des suivis n'est pas attestée. Les travaux américains insistent sur le manque de suivi et d'évaluation (Kondolf et Micheli, 1995) et concluent pour certains à la dissociation des deux (Bernhardt et al., 2007).

\section{Un suivi davantage développé pour certaines actions}

Le suivi, s'il n'est pas développé uniformément en fonction des types de restauration, varie également suivant la nature des mesures. L'analyse du développement des suivis postrestauration relativement aux mesures de restauration écologiques et piscicoles est sur ce point significative (Fig. 5). En valeur absolue, ce sont les mesures le plus souvent mises en œuvre qui sont le plus souvent suivies (aménagements piscicoles et reconnexions d'annexes). Pourtant, en valeurs relatives, les actions plus originales, qu'on pourrait considérer comme pilotes, sont bien logiquement mieux évaluées (exemple des relèvements des débits). Viennent ensuite les travaux de recharge sédimentaire ou de restauration piscicole par réintroduction de substrats, qui sont respectivement suivis à hauteur de $50 \%$ et $64 \%$. À l'inverse, seules $24 \%$, $18 \%$ et $40 \%$ des restaurations d'habitats, de frayères ou d'annexes sont suivies. Enfin, les opérations les plus exemplaires sont souvent accompagnées par des structures scientifiques et font l'objet de financements. C'est le cas pour le relèvement des débits sur le Rhône ou pour les travaux de recharges sédimentaires de l'Ain ou de la Drôme.

\section{L'utilisation des indicateurs et des paramètres d'évaluation}

L'efficacité de l'évaluation ne dépend pas seulement du nombre d'éléments de suivi développés, mais aussi de 


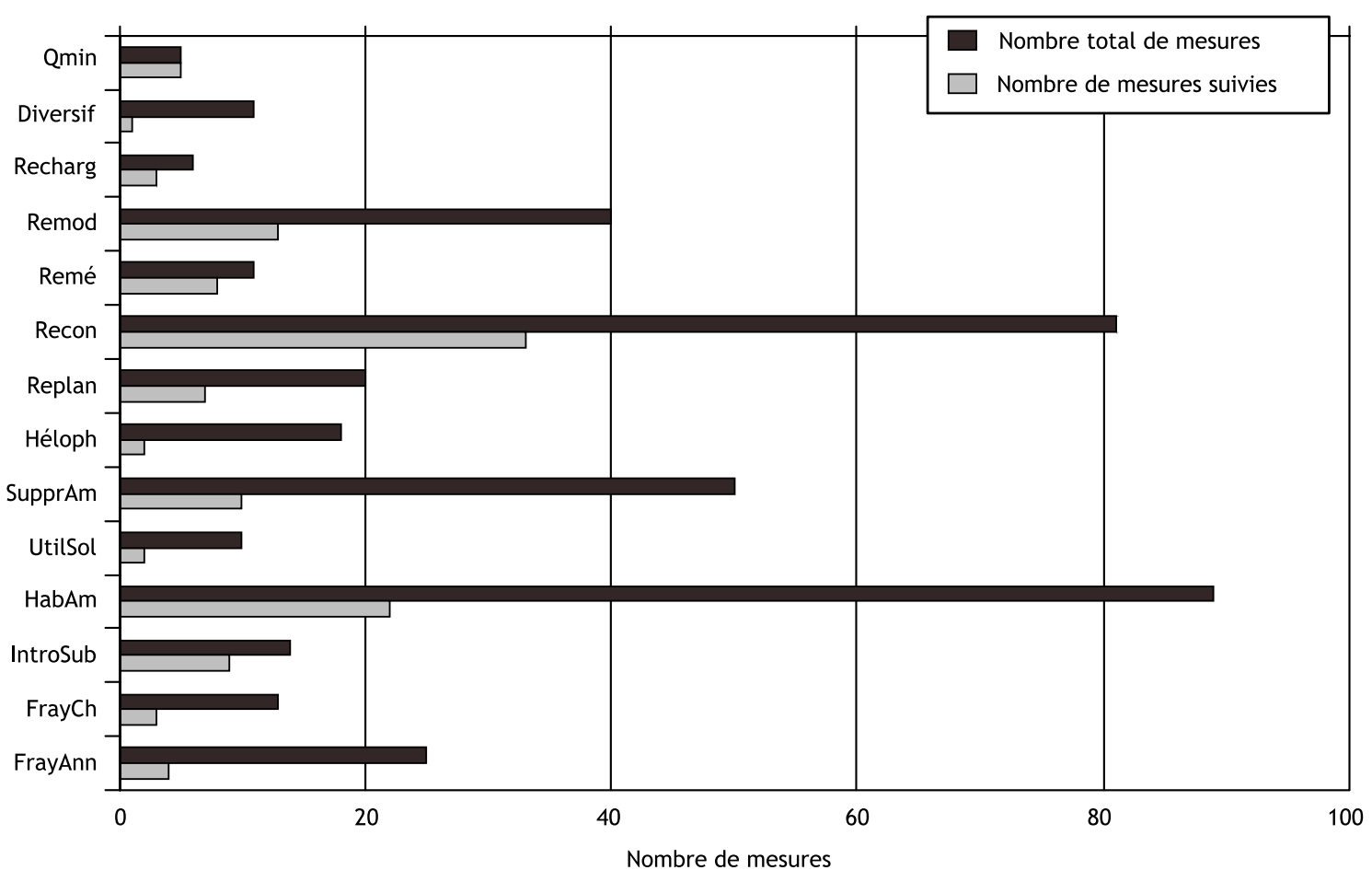

Fig. 5. Nombre de mesures pour lesquelles un suivi postrestauration a été développé en fonction du nombre total de mesures (voir Tab. 2 pour les codes).

la manière dont ils sont construits. Il est alors déterminant de s'intéresser à la nature des métriques utilisées pour la définition du succès ou de l'échec de l'action (Bazin et Barnaud, 2002; Woolsey et al., 2007). Un premier constat réside dans la confusion entre les paramètres et les indicateurs. Beaucoup de suivis sont fondés sur de simples paramètres, parfois très empiriques, correspondant à des relevés visuels de terrain. L'utilisation de véritables indicateurs est plus rare. Sur les 95 opérations ayant fait l'objet de suivis postrestauration déclarés, seulement 21 mobilisent au moins un indicateur alors que 39 mobilisent des paramètres. Il faut noter que $47 \%$ des opérateurs déclarant un élément de suivi ne détaillent pas les paramètres ou indicateurs utilisés. On peut alors s'interroger sur la fiabilité des évaluations produites.

Si l'on s'intéresse ensuite à la nature des paramètres et indicateurs, on observe une nette apparition de certaines tendances. Alors que la littérature scientifique insiste sur la diversité des outils à disposition, on constate que les gestionnaires mobilisent souvent les mêmes, à savoir : l'indice biologique global normalisé, l'indice poisson rivière et l'indice d'attractivité morphodynamique. Cela peut sembler positif dans une réflexion sur la définition de protocoles généralisables; mais on peut aussi se demander dans quelle mesure l'utilisation prend en compte la spécificité de l'action. Par ailleurs, les évaluations sont centrées sur les bénéfices écologiques, alors que les questions d'acceptation et de bénéfices sociaux, pourtant déterminantes (Piégay et al., 2009), sont rarement abordées directement. Le débat sur la question des objectifs est ouvert. Pourquoi restaure-t-on? L'absence de définition explicite des objectifs est frappante : ces derniers sont formulés dans seulement $49 \%$ des actions. Restaure-t-on uniquement pour répondre à des dégradations? Les résultats montrent que $71 \%$ des actions ne répondent à aucun dysfonctionnement déclaré. Il faut alors chercher d'autres raisons à la mise en œuvre de la restauration.

\section{Restauration entre action et communication}

\section{Qui agit et qui communique sur Internet?}

L'utilisation du médium Internet permet d'étudier les mécanismes d'action et de communication. Il faut alors établir une distinction entre les structures qui portent les restaurations (les maîtres d'ouvrages), celles qui les soutiennent (techniquement, scientifiquement ou financièrement) et celles qui communiquent sur ces opérations (Fig. 6). On constate que les structures en charge des outils d'intervention (syndicats notamment) sont celles qui agissent le plus (224 actions) et qui communiquent à hauteur de leurs activités. À l'inverse, les structures compétentes dans le domaine de la pêche, qui sont les deuxièmes plus actives, communiquent plus qu'elles n'agissent (129 actions contre 77), écart toutefois 


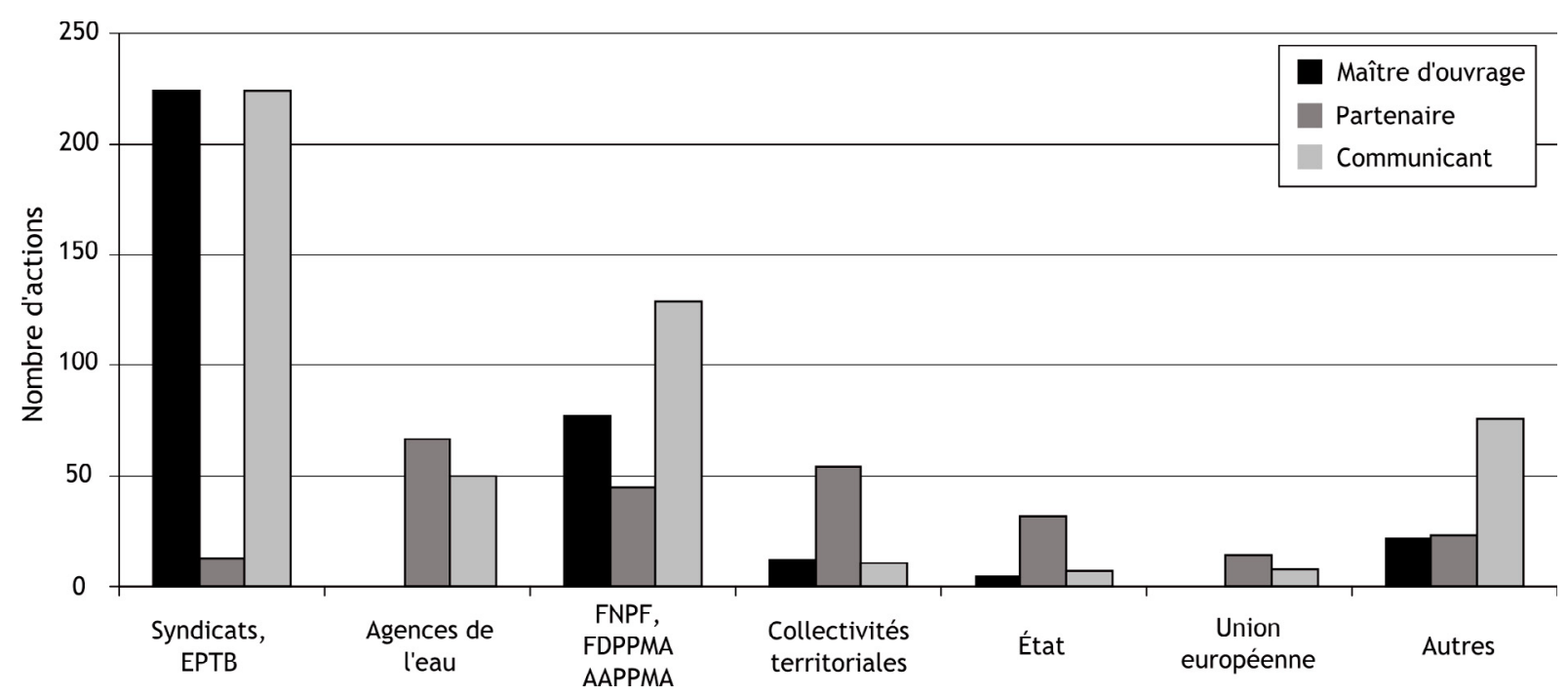

Fig. 6. Implication des structures en fonction de leur statut.

compensé par leur implication partenariale (45 actions). Les structures administratives (agences de l'eau, collectivités territoriales, État) constituent essentiellement des soutiens à l'action (153 actions), bien qu'elles portent aussi 17 projets. Une place particulière doit être accordée aux soutiens européens, généralement dans le cadre de programmes LIFE (par exemple LIFE Ruisseaux, Basse vallée de l'Ain, Eau et forêt). Il s'agit d'aides financières qui sont à distinguer des appuis "médiatiques ". Les agences de l'eau, par exemple, soutiennent 67 actions et en mentionnent 50. Il y a également une activité Internet accentuée de la part des acteurs privés et associatifs (76 actions mentionnées), qui répond à des logiques plurielles : militantisme écologique, publicité environnementale, etc. En valeur absolue, ce sont toutefois les structures de terrain (syndicats, AAPPMA, FDPPMA. . .) qui communiquent le plus. Elles disposent pour cela de plateformes Internet qui facilitent la diffusion de l'information, alors que les autres acteurs disposent d'espaces souvent isolés. Le travail de recensement a d'ailleurs privilégié les sites Internet des grands opérateurs nationaux.

\section{Qui communique sur quoi?}

S'il est important de déterminer à quelle hauteur les structures agissent et communiquent, l'analyse doit également porter sur le rapport entre implication des structures et nature des opérations, afin d'identifier des dynamiques d'intervention. En prenant une typologie fondée sur les trois types de restauration identifiés, on se rend compte que les opérateurs mobilisés changent d'une conception de la restauration à l'autre. D'une comparaison entre entités qui agissent et entités qui communiquent, on est passé à une distinction entre entités impliquées dans des actions écologiques, piscicoles ou hydrauliques (Fig. 7). La plupart des résultats concordent alors avec les compétences, les échelles d'intervention et les niveaux d'investissement propres à chaque opérateur. Les structures de pêche se déclarent impliquées majoritairement dans des actions à objectif piscicole et, en second lieu, écologique. Seules, elles privilégient la mise en œuvre de mesures tels la diversification des écoulements, l'aménagement d'habitats et la restauration de frayères. Au niveau des structures syndicales, les résultats traduisent des conceptions plus hétérogènes. Elles sont impliquées dans des politiques de gestion hydraulique et communiquent sur ce type d'actions. Ce sont des mesures relatives à la sécurité des biens et des personnes qu'elles appliquent prioritairement quand elles agissent seules : entretien de chenal et de ripisylve; protection de berges; gestion hydraulique. La restauration écologique ne semble donc pas prédominante dans la mise en œuvre des SAGE et des CR et la distinction entre les différentes actions est souvent fine. Au niveau des collectivités, l'implication est aussi plurielle. Elle traduit peut-être une dépendance plus importante aux besoins sociétaux, en termes de naturalité ou de sécurité. Cette ambiguité se retrouve peu en ce qui concerne les structures privées qui communiquent beaucoup sur les actions écologiques, même si leurs motivations diffèrent. Le contexte socioenvironnemental actuel peut constituer un facteur explicatif. Plus surprenante est la position des agences de l'eau, qui, bien que fortement impliquées dans les actions écologiques, ne continuent pas moins à soutenir des travaux hydrauliques. Ce constat ne peut que renforcer les inquiétudes actuelles concernant les réponses que les opérateurs peuvent apporter aux exigences de la DCE et à la conciliation des deux types d'action. Les financements européens sont débloqués essentiellement et, sans surprise, au profit d'opérations écologiques, mais 


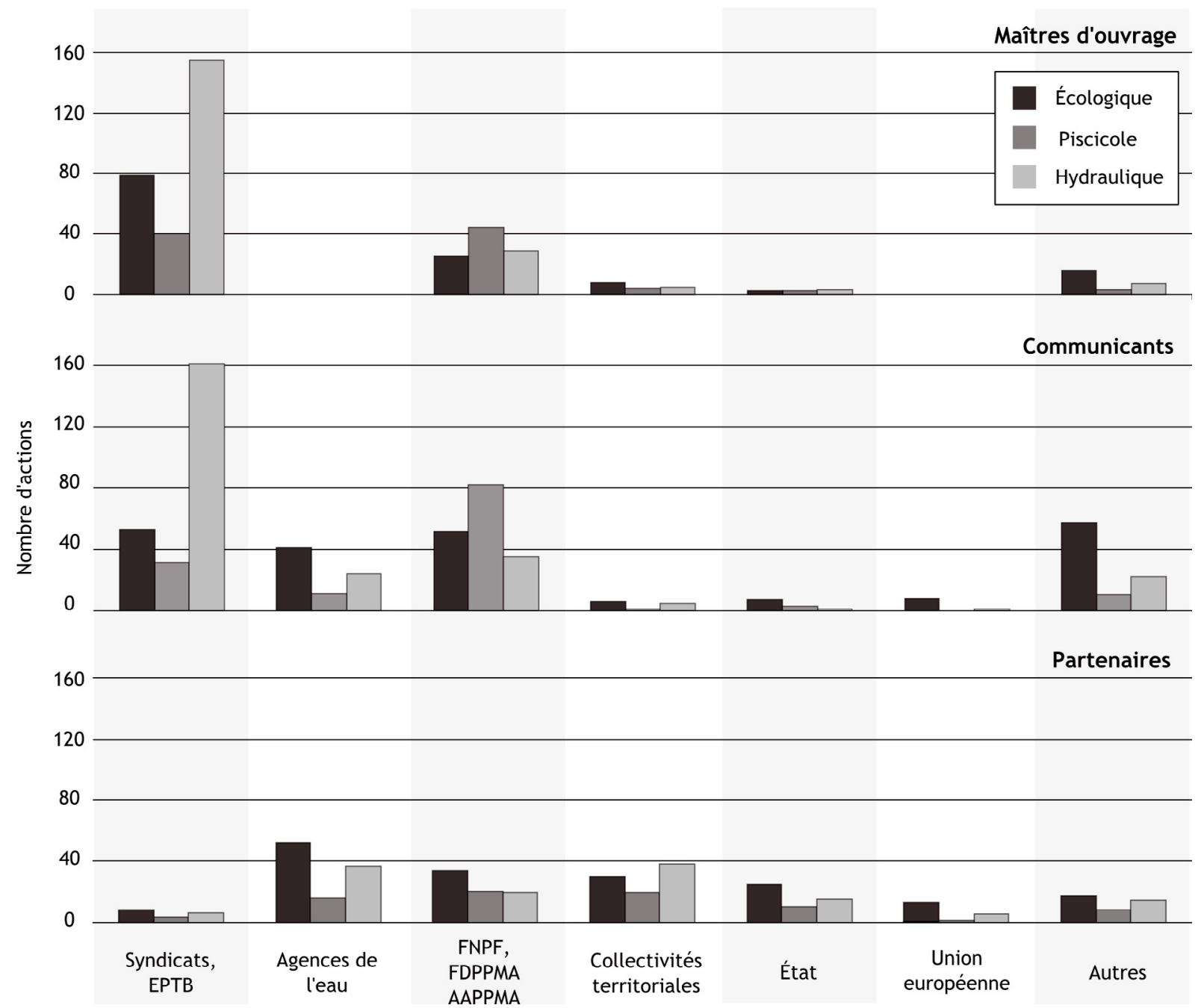

Fig. 7. Implication des structures en fonction de leur statut et du type de restauration.

l'Europe apporte son soutien également au niveau hydraulique, témoignant sans doute d'une volonté d'approche plus globale.

\section{Conclusions et perspectives}

Ces résultats permettent d'établir un premier bilan en matière de restauration de rivières. L'intérêt est scientifique et opérationnel, constituant une base de réflexion conceptuelle et une source de retours d'expériences pour la mise en œuvre des actions futures. Cependant, le travail n'est pas sans limites et ne saurait en aucun cas prétendre à l'exhaustivité, compte tenu notamment de la nature de la méthode utilisée. Dans quelle mesure cette synthèse des données présentes sur Internet est-elle représentative de la réalité des actions françaises? Deux axes de travail sont aujourd'hui développés pour apporter des éléments de réponse. Tout d'abord, une étude de cas, fondée sur des entretiens téléphoniques, est en cours sur des opérations considérées comme exemplaires. Elle pourra être mise en perspective des avancées internationales de la recherche à travers une analyse des publications scientifiques. Ensuite, un travail devrait être conduit sur l'emploi des termes dans les guides techniques de gestion des milieux aquatiques et dans les demandes de financements formulées auprès des autorités compétentes. Le présent recensement constitue dans ce cadre une étape marquante. Il retraduit en effet les tendances nationales et permet de valider ou d'invalider les hypothèses initiales. Plusieurs remarques conclusives peuvent alors être formulées.

Si la mise en place des structures et des outils d'intervention joue un rôle certain dans la réalisation des actions, elle ne saurait suffire et ne peut constituer un achèvement en soi. D'autres mécanismes interviennent dans l'explication de la réalisation d'un projet de restauration. Le manque courant de distinction entre la restauration écologique et la restauration ou l'entretien hydraulique est sans doute l'expression la plus significative du travail conceptuel et sémantique à réaliser, ne serait-ce que pour 
répondre aux objectifs de la DCE. La restauration renvoie généralement à trois définitions : une dimension hydraulique et ingénieriste centrée sur des enjeux sécuritaires ; une approche piscicole soucieuse d'une ressource ; une conception écologique considérant le bon fonctionnement comme le garant d'un développement durable. Cette segmentation conduit à s'interroger sur la réalité de la politique de gestion intégrée des bassins versants.

La différence de conceptions s'exprime géographiquement. Toutefois, si la répartition est claire, elle reste encore à comprendre. Des éléments d'explication peuvent être proposés, comme l'importance des structures d'encadrement, les politiques d'intervention de certaines agences de l'eau ou les différences régionales en matière de perception environnementale, mais ils constituent autant d'hypothèses à vérifier. Le nombre d'actions en région Rhône-Alpes ne peut, par exemple, s'expliquer par le simple maillage des structures de gestion. À l'inverse, la précocité d'une conscience environnementale dans l'Est est bien notable et semble dépasser la seule région d'Alsace.

Si des différenciations spatiales ont pu être identifiées à l'échelle nationale, aucune évolution temporelle des conceptions n'est notable à partir des informations communiquées sur Internet. Certes, l'émergence du génie végétal dans les années 1990 et l'augmentation significative des mesures écologiques en 2000 ne sont pas anecdotiques, mais le terme de restauration reste employé selon une acception élargie, différente de celle de la DCE. La pertinence des politiques en matière de restauration peut alors être interrogée. La distinction est-elle notamment bien établie dans la destination des financements?

On ne peut que souligner les lacunes de l'information disponible sur Internet relative au suivi et à l'évaluation des actions réalisées, et ce, quelle que soit l'échelle de travail. L'absence de recensement national est significative en elle-même. La réflexion sur l'impact de politiques et de structures compétentes dans le domaine est à construire. Le manque s'exprime également, à l'échelle du bassin versant ou du site restauré, dans le suivi de l'action en propre. Les travaux réalisés ont-ils des répercussions positives sur le milieu? Quelle est la pertinence des actions? Quels sont les gains écologiques et socioéconomiques? Alors que les actions se multiplient, il est nécessaire d'apporter des réponses : scientifiquement, pour faire progresser la connaissance dans le domaine ; opérationnellement, pour ne pas reconduire des actions vouées à l'échec et répondre aux exigences de la DCE ; ou encore économiquement, pour justifier l'importance des financements accordés. De fait, notre quatrième hypothèse n'est pas vérifiée : les acteurs de la restauration ne communiquent pas véritablement d'informations sur la réussite ou non des opérations.

La communication via Internet est en augmentation, ce qui atteste d'une préoccupation croissante pour les questions de restauration de rivières. Elle répond bien entendu aux exigences nationales et européennes, mais aussi peut-être à des demandes sociales de plus en plus marquées par les préoccupations environnementales. S'il y a diffusion de l'information, il y a également une demande de la part des opérateurs. Ils travaillent souvent de manière coordonnée lorsqu'il s'agit d'opérations de restauration écologique et les communicants sont d'abord les maîtres d'ouvrage.

\section{Remerciements}

Ces travaux s'inscrivent dans le cadre de la zone atelier Bassin du Rhône et du projet européen IWRM/Net FORECASTER (2008-2010). Les auteurs remercient les coordonnateurs de ce programme, en particulier N. Lamouroux, et les différents opérateurs avec qui des discussions fructueuses ont été établies, en particulier S. Stroffek et L. Bourdin, de l'agence de l'eau RM\&C, ainsi que V. Nicolas, J. Péress, C. Barnetche, J.-M. Baudouin, C. Forst et F. Weingertner, de l'ONEMA. Les auteurs remercient également les relecteurs de cet article pour leurs corrections et leurs suggestions.

\section{Références}

Andrieu, O., 1998. Trouver l'info sur l'Internet, Paris, Eyrolles.

Barnaud, G., 1995. À l'interface de la pratique et de la théorie : l'écologie de la restauration, Natures Sciences Sociétés, horssérie « Recréer la nature », 36-50.

Bash, J.S., Ryan, C.M., 2002. Stream restoration and enhancement projects: Is anyone monitoring?, Environmental Management, 29, 6, 877-885.

Bazin, P., Barnaud, G., 2002. Du suivi à l'évaluation : à la recherche d'indicateurs opérationnels en écologie de la restauration, La Terre et la Vie : revue d'écologie, supplément 9, 201-224.

Bernhardt, E.S., Sudduth, E.B., Palmer, M.A., Allan, J.D., Meyer, J.L., Alexander, G., Follastad-Shah, J., Hassett, B., Jenkinson, R., Lave, R., Rumps, J., Pagano, L., 2007. Restoring rivers one reach at a time: Results from a survey of U.S. river restoration practitioners, Restoration Ecology, 15, 3, 482-493.

Boni, A., Stemart, N., 2008. Google, Paris, Micro Application.

Bradshaw, A.D., 1996. Underlying principles of restoration, Canadian Journal of Fisheries and Aquatic Sciences, 53, Supp. 1, 3-9.

Cairns, J., Jr., Heckman, J.R., 1996. Restoration ecology: The state of an emerging field, Annual Review of Energy and the Environment, 21, 167-89.

Dufour, S., Piégay, H., 2009. From the myth of a lost paradise to targeted river restoration: Forget natural references and focus on human benefits, River Research and Applications, 25, 5, 568-581.

Habersack, H., Piégay, H., 2007. River restoration in the Alps and their surroundings: Past experience and future challenges, in Habersack, H., Piégay, H., Rinaldi, M. (Eds), Gravel-Bed Rivers 6: From Process Understanding to the Restoration of Mountain Rivers. Developments in Earth Surface Processes, Amsterdam \& London, Elsevier B.V., 703-708. 
Jenkinson, R.G., Barnas, K.A., Braatne, J.H., Bernhardt, E.S., Palmer, M.A., Allan, J.D., The National River Restoration Science Synthesis, 2006. Stream restoration databases and case studies: A guide to information resources and their utility in advancing the science and practice of restoration, Restoration Ecology, 14, 2, 177-186.

Kondolf, G.M., Micheli, E.R., 1995. Evaluating stream restoration projects, Environmental Management, 19, 1, 1-15.

Kondolf, G.M., Anderson, S., Lave, R., Pagano, L., Merenlender, A., Bernhardt, E.S., 2007. Two decades of river restoration in California: What can we learn?, Restoration Ecology, 15, 3, 516-523.

Lachat, B., Adam, P., Frossard, P.-A., Marcaud, R., 1994. Guide de protection des berges de cours d'eau en techniques végétales, Paris, Ministère de l'Aménagement du territoire et de l'Environnement, DIREN Rhône-Alpes.

Le Floc'h, É., Aronson, J., 1995. Écologie de la restauration : définition de quelques concepts de base, Natures Sciences Sociétés, hors-série "Recréer la nature », 29-35.

Le Lay, Y.-F., Piégay, H., Gregory, K., Chin, A., Dolédec, S., Elosegi, A., Mutz, M., Wyzga, B., Zawiejska, J., 2008. Variations in cross-cultural perception of riverscapes in relation to in-channel wood, Transactions of the Institute of British Geographers, 33, 2, 268-287.

Malavoi, J.-R., Adam, P., 2007. La restauration hydromorphologique des cours d'eau : concepts et principes de mise en œuvre, Ingénieries-EAT, 50, 49-61.
Maniez, D., 2008. Les Dix Plaies d'Internet : les dangers d'un outil fabuleux, Paris, Dunod.

National Research Council (NRC), 1992. Restoration of Aquatic Ecosystems, Washington (DC), Academy Press.

Palmer, M.A., Bernhardt, E.S., 2006. Hydroecology and river restoration: Ripe for research an synthesis, Water Resources Research, 42, W03S07.

Piégay, H., Bornette, G., Lamouroux, N., 2009. Geomorphology and river restoration feedback and outstanding issue in the hydrographic district of the Rhône river. River restoration as a collaboration with nature, in Gumiero, B., Rinaldi, M., Fokkens, B. (Eds), Proceedings: IVth ECRR International Conference on River Restoration 2008, Venice, San Servolo Island, 16-19 June 2008, ECRR/CIRF, Venice Editor, 67-82.

Roni, P., Hanson, K., Beechie, T., Pess, G., Pollock, M., Bartley, D.M., 2005. Habitat Rehabilitation for Inland Fisheries: Global Review of Effectiveness and Guidance for Rehabilitation of Freshwater Ecosystems. FAO Fisheries Technical Paper, No. 484. FAO, Rome.

Sachon, G., Ahyerre, M., Wasson, J.-G., 2003. Diagnostic et restauration des milieux aquatiques pour 2015. Quelques questions soulevées par la directive-cadre européenne sur l'eau, Techniques Sciences Méthodes, 1, 39-44.

Woolsey, S., Capelli, F., Gonser, T., Hoehn, E., Hostmann, M., Junker, B., Paetzolds, A., Roulier, C., Schweizer, S., Tiegs, S.D., Tockner, K., Weber, C., Peter, A., 2007. A strategy to assess river restoration success, Freshwater Biology, 52, 752-769.

Reçu le 29 janvier 2010. Accepté le 6 janvier 2011. 